\title{
Psychological changes in alcohol-dependent patients during a residential rehabilitation program
}

This article was published in the following Dove Press journal:

Neuropsychiatric Disease and Treatment

7 December 2015

Number of times this article has been viewed

Ines Giorgi'

Marcella Ottonello 2,3

Giovanni Vittadini ${ }^{4}$

Giorgio Bertolotti ${ }^{5}$

'Psychology Unit, Salvatore Maugeri Foundation, Clinica del Lavoro e della Riabilitazione, IRCCS,

Pavia, ${ }^{2}$ Department of Physical \& Rehabilitation Medicine, Salvatore Maugeri Foundation, Clinica del Lavoro e della Riabilitazione, IRCCS, Genoa, ${ }^{3}$ Department of Medicine, $\mathrm{PhD}$ Program in Advanced Sciences and Technologies in Rehabilitation Medicine and Sport, Università di Tor Vergata, Rome, ${ }^{4} \mathrm{Alcohol}$ Rehabilitation Unit, Salvatore Maugeri Foundation, Clinica del Lavoro e della Riabilitazione, IRCCS, Pavia, ${ }^{5}$ Psychology Unit, Salvatore Maugeri Foundation, Clinica del Lavoro e della Riabilitazione, IRCCS, Tradate, Italy

Correspondence: Marcella Ottonello Department of Physical \& Rehabilitation Medicine, Salvatore Maugeri Foundation, Clinica del Lavoro e della Riabilitazione, IRCCS, 14 via Missolungi, Nervi, Genoa 16167, Italy

Fax +39010 30791269

Email marcella.ottonello@fsm.it
Background: Alcohol-dependent patients usually experience negative affects under the influence of alcohol, and these affective symptoms have been shown to decrease as a result of alcohol-withdrawal treatment. A recent cognitive-affective model suggests an interaction between drug motivation and affective symptoms. The aim of this multicenter study was to evaluate the psychological changes in subjects undergoing a residential rehabilitation program specifically designed for alcohol addiction, and to identify at discharge patients with greater affective symptoms and therefore more at risk of relapse.

Materials and methods: The sample included 560 subjects (mean age $46.91 \pm 10.2$ years) who completed 28-day rehabilitation programs for alcohol addiction, following a tailored routine characterized by short duration and high intensity of medical and psychotherapeutic treatment. The psychological clinical profiles of anxiety, depression, psychological distress, psychological well-being, and self-perception of a positive change were assessed using the Cognitive Behavioral Assessment - Outcome Evaluation questionnaire at the beginning and at the end of the program. The changes in the psychological variables of the questionnaire were identified and considered as outcome evaluation of the residential intervention. Moreover, differences in the psychological functioning between patients with different characteristics were investigated.

Results: The score measured by the Cognitive Behavioral Assessment - Outcome Evaluation showed significant improvements in all the psychological characteristics assessed, and the profile at discharge was within the normal scores. Some significant differences were found in relation to specific characteristics of the sample, such as age, sex, level of education, type of intervention, and polysubstance use.

Conclusion: This study shows the changes in psychological profile in subjects undergoing residential rehabilitation from alcohol and how this profile may permit identification of subjects requiring more psychosocial support after discharge.

Keywords: alcohol dependence, alcohol withdrawal, outcome measure, residential rehabilitation, psychological support

\section{Introduction}

Alcohol dependence causes physical and emotional problems and has a huge impact on family life, employment, violence, and crime. ${ }^{1-3}$ Families and society are disrupted. Abuse of alcohol affects nearly every bodily system and increases the risk of certain types of cancer (ie, oral, pharyngeal, liver, and lung cancers). ${ }^{4,5}$

Treatment for alcoholism can be performed either within an inpatient hospital setting or as an outpatient program. ${ }^{6,7}$ Inpatient rehabilitation 28-day programmes ${ }^{8}$ provide highly structured treatments, including group therapy, individual therapy, and alcoholism-education sessions designed to address the specific behavior of drinking with information feedback, health education, skill building, and practical advice (alcohol-education materials). Furthermore, professional staff members are available 
around the clock to help manage the patient's acute medical and psychological problems during the initial treatment period (ie, detoxification).

Over the last 30 years, there has been a gradual increase of residential rehabilitation programs for alcohol disorders in Italy. The therapeutic approach is inspired by both American experience of the Minnesota Model, ${ }^{9}$ Croatian psychiatry studies, ${ }^{10}$ and finally by German and Austrian "psychosomatic clinics". ${ }^{11}$ All the six rehabilitation centers who participated in the present study are members of the CORRAL (COordinamento of Residenzialità Riabilitative ALcologiche) association, and adhered to the same rehabilitation model. Specific features of this model are: ${ }^{8}$

1. Shortness of hospitalization (28 days)

2. Intensity of intervention (physical, psychic, and family)

3. Complexity of intervention itself(with provision of medical services, psychological, and educational)

4. Elaboration of an aftercare project.

The aim of this multicenter study was to evaluate the residential program in terms of psychological functioning of inpatients addicted to alcohol. Furthermore, among the numerous mechanisms that may underlie craving, a recent cognitive-affective mode ${ }^{12}$ suggested an interaction between drug motivation and affective symptoms. They stated the importance of negative reinforcement as the principal motive for drug use, where drinking is used as a means to escape negative affect. More recent research ${ }^{13}$ has also highlighted an increased level of anxiety and alcohol craving in the early recovery from alcoholism, particularly with exposure to stressful or alcohol-related stimuli. We wished also to investigate the possible differences in the psychological variables through the examination of specific characteristics (ie, age, sex, education, type of dependence, type of intervention, and polysubstance use) within the study sample, and to assess at discharge subjects with higher affective symptoms. The objective was to examine associations between sociodemographic factors and severity of alcohol dependence and psychiatric symptoms among individuals in a detoxification program.

Alcohol withdrawal is an important initial step in the treatment of alcohol dependence, and the evaluation of changes in psychological symptoms, as well as anxiety, depression, and psychological distress allow identification at discharge of patients who need more care in mental health community services.

\section{Materials and methods}

\section{Patients}

The sample comprised 560 hospitalized patients who had completed the rehabilitation program, from 584 patients consecutively admitted to the six rehabilitation centers over a period of 1 year: 417 men (74\%) and 143 (26\%) women. Their ages ranged from 20 to 75 (mean 46.91, standard deviation 10.2) years. All participants had a diagnosis of alcohol dependence, as defined by the Diagnostic and Statistical Manual of Mental Disorders. ${ }^{14}$ Of these, $42.5 \%$ had a history of 10 or more years of alcohol dependence, and only $9.8 \%$ had a history of less than 5 years of addiction. Participants reported a desire to stop drinking and a willingness to undergo an intensive therapeutic program characterized by a short duration and a high intensity of medical and psychotherapeutic treatment. Approximately half (54.8\%) of the subjects also had addictions to other substances (cannabis, cocaine, benzodiazepines). Comorbid psychiatric disorders were personality disorder (41\%), affective syndrome (28\%), anxiety syndrome (12\%), and psychosis (4\%). The Scientific and Technical Committee of the Care and Research Institute of Tradate (Varese, Italy), Salvatore Maugeri Foundation IRCCS, discussed and approved the study on July 11, 2014.

\section{Procedure}

All rehabilitation centers involved in the study adhered to the same rehabilitation model (Zambon et $\mathrm{al}^{8}$ ), characterized by short duration and a complex, intense therapeutic intervention mainly addressed at patients with a severe clinical condition and low level of social problems. In particular, this rehabilitative intervention constitutes:

- A multiprofessional intervention (medical, nursing, psychological, educational)

- Evaluation and treatment of acute withdrawal symptoms

- Group therapy to support and maintain the motivation to change, to educate on alcohol-related risks, and to promote the activation of interpersonal skills

- Group activities with various modes of expression, such as movie discussions, gymnastics, theme-based groups (ie, managing emotions, self-esteem promotion)

- Personal involvement in the care of the patients, as well as taking care of themselves and their environment (ie, keeping their room tidy, making their bed)

- Group meetings with family members (if available) with functions of support, counseling, and involvement in the rehabilitation program.

Following admission to the rehabilitation unit, the patients received an initial evaluation by an addiction-medicine physician or psychiatrist with wide experience in managing addiction. This evaluation served to guide medical management during detoxification and discharge planning. Psychosocial assessments were also conducted together with the local mental health services to arrange for appropriate outpatient 
treatment aftercare. During residential rehabilitation, patients were required to attend 1-hour group-therapy sessions twice daily regarding maintenance of abstinence, education of alcohol-related risks, motivation to change, and relapse prevention. The afternoon sessions included the group activities (movie discussions, gymnastics, relaxation techniques, and art therapy). Family sessions were held once a week.

A written informed-consent form was issued to the patients informing them of the study and to obtain their permission to use the data resulting from the program, and written informedconsent was obtained for all participants. Through a short interview, conducted by the physician of the ward, personal information, such as age, level of education, work status, period of alcohol dependence, possible presence of other drug dependence, or psychiatric comorbidities, was collected in the initial assessment performed on admission. The "intervention" variable was defined as two types of intervention: psychotherapy with or without pharmacotherapy. The patients that received each of the two interventions were those that had already used drugs for their mental health symptoms or required drugs for symptoms during hospitalization. The demographic characteristics of the sample population are highlighted in Table 1. Later, a self-administered questionnaire - the Cognitive Behavioral Assessment - Outcome Evaluation

Table I Characteristics of the studied groups

\begin{tabular}{ll}
\hline Variable & $\mathbf{n}$ \\
\hline Sex & \\
Male & $417(74.4 \%)$ \\
Female & $143(25.5 \%)$ \\
Level of education & \\
$\quad$ Years $\leq 8$ & $331(59.1 \%)$ \\
$8<$ Years $<13$ & $159(28.4 \%)$ \\
Years $>$ I3 & $42(7.5 \%)$ \\
Missing values & $28(5 \%)$ \\
Employment status & \\
Employed & $265(47.3 \%)$ \\
Unemployed & $181(32.3 \%)$ \\
Retired & $57(10.2 \%)$ \\
Other & $27(4.8 \%)$ \\
Missing values & $30(5.4 \%)$ \\
Period of alcohol dependence & \\
Years $\leq 5$ & $55(9.8 \%)$ \\
$5<$ Years $<10$ & $95(17 \%)$ \\
Years $\geq 10$ & $238(42.5 \%)$ \\
Missing values & $172(30.7 \%)$ \\
Substance dependence & \\
Pure alcoholics & $253(45.1 \%)$ \\
Polyabusers & $307(54.8 \%)$ \\
Intervention & \\
Pharmaco- + psychotherapy & $338(60.3 \%)$ \\
Psychotherapy & $211(37.6 \%)$ \\
Missing values & $11(1.9 \%)$ \\
Age (years), mean \pm standard deviation & $46.91 \pm 10.2$ \\
\hline
\end{tabular}

$(\mathrm{CBA}-\mathrm{OE})^{15}$ - was delivered to evaluate psychological functioning at admission and at the end of rehabilitation. All data collected in this study were derived from tools and treatments used in routine clinical practice of the centers involved, and were analyzed by independent clinicians. The institutional review board (Comitato Tecnico Scientifico, Maugeri Foundation, Institute of Tradate) approved the retrospective study. A standard interview was used to ascertain alcohol consumption at 6 months after discharge of subjects who had completed the residential rehabilitation program. Follow-up interviews were conducted over the phone or by email survey by a nurse.

\section{Measures}

The CBA-OE was the questionnaire used to evaluate psychological treatment effects during the residential program. The questionnaire has 80 items and uses a 5-point scale, ranging from $1=$ nothing to $5=\mathrm{a}$ lot. The $\mathrm{CBA}-\mathrm{OE}$ is articulated over the following five scales: anxiety (14 items, eg, "I have been upset about trivial things"); well-being (15 items, eg, "I have done things that interested and involved me"); perception of positive change, of getting others' support, and being able to cope with difficulties (eleven items, eg, "I have tried to deal with difficulties rather than avoid them"); depression (19 items, eg, "I have been tormented by feelings of guilt"); psychological distress or serious symptoms of disorder and poor control over impulses (21 items, eg, "I have felt debased or mocked"). Instructions for the CBA-OE require participants to answer each item by referring to the previous 2 weeks. The questionnaire has shown excellent psychometric properties: it has a strong factor structure, good reliability, satisfactory criterion-related and convergent validity, and has the power to discriminate among nonclinical and "suffering-distressed" subjects. ${ }^{15,16}$ Cronbach's $\alpha$ was between 0.80 and 0.91 for the clinical sample and between 0.74 and 0.91 in the nonclinical sample. The self-administered questionnaire was compiled on admission to the rehabilitation program and again 3 days before discharge.

\section{Data analysis}

We used a descriptive analysis (percentage, median, and medium score) to present the basic characteristics of the study participants (Table 1). In the present study, we identified within the group of alcoholics in treatment some specific patient categories. These subgroups were formed on the basis of individual characteristics. The patients were stratified into groups by sex, level of education, work status (employed, unemployed, retired), substance dependence (pure abusers of alcohol vs polyabusers), type of intervention (drugs plus psychotherapy sessions vs psychotherapy sessions only). We investigated possible differences in 
psychological variables evaluated by the CBA-OE using the Mann-Whitney $U$-test or Kruskal-Wallis test (in the case of more than two groups of variables) to match nonparametric variables, since the questionnaire is an ordinal scale of measurement. We also assumed that the data was not normally distributed. Bonferroni correction was used for multiple comparisons. The significance in CBA-OE score changes between admission and discharge was tested using the Wilcoxon matched-pairs signed-rank test (nondirectional, $\alpha=0.05)$. The size effect was also calculated using Cohen's d-method. In his authoritative Statistical Power Analysis for the Behavioral Sciences, Cohen (1988) outlined a number of criteria for gauging small, medium, and large effect sizes in different metrics: d-effects - small $\geq 0.20$, medium $\geq 0.50$, large $\geq 0.80 .{ }^{17}$ Correlations with the variable of age were calculated as Spearman's $\rho$, corrected for ties. The interviews conducted at 6-month follow-up provided only a qualitative analysis on alcohol consumption.

\section{Results}

Descriptive statistics for the whole study sample are shown in Table 1. The mean score, median, and the interquartile range of $\mathrm{CBA}-\mathrm{OE}$ scales at admission and at discharge are shown in Table 2. In Table 3, the mean scores and standard deviation in the variables of sex, type of dependency, education, and intervention are indicated.

Changes in CBA-OE scales between the admission and the discharge of the rehabilitation program are compared in Table 4. We found significant differences in relation to the sex variable at admission on the scales of anxiety $(P<0.001)$, well-being $(P<0.001)$, positive changes $(P<0.05)$, and depression $(P<0.001)$, and only on the scale of well-being $(P<0.05)$ at discharge. Females had higher anxiety and depression scores than males, while the scores on wellbeing and positive changes were lower. Significant differences between levels of education were found on the scale of psychological distress $(P<0.05)$ between primary and secondary school and high school at admission and discharge, and only at discharge on the scales of anxiety $(P<0.01)$ and depression $(P<0.05)$. A significant difference between high school and university on the scale of well-being $(P<0.05)$ was seen at discharge.

There were differences between the "pure abuser" and "polyabuser" groups on all five scales of the CBA-OE at admission (respectively, $P<0.0001, P<0.05, P<0.05$, $P<0.0001$, and $P<0.0005)$ and only on the scales of anxiety $(P<0.0001)$, depression $(P<0.001)$, and psychological distress $(P<0.001)$ at discharge. On the intervention variable, we found differences between groups on all the scales of CBA-OE at admission $(P<0.0001)$ and at discharge on the anxiety $(P<0.001)$, depression $(P<0.05)$, and psychological distress scales $(P<0.001)$, as in the previous substance-dependence variable. With regard to the employment status variable, we found significant differences in the scale of psychological distress at admission between groups $(P<0.008)$, and at discharge we found differences in the scales of positive change and well-being (respectively, $P<0.0002$ and $P<0.009$ ).

Finally, we found a significantly negative correlation between the age variable and the anxiety $(P<0.0001)$, depression $(P<0.01)$, and psychological distress scales $(P<0.0001)$ of the CBA-OE at admission, and at discharge the correlation was on the scales of anxiety $(P<0.005)$, well-being $(P<0.05)$, positive change $(P<0.05)$, and psychological distress $(P<0.0005)$. The Intervention column in Table 3 shows a significant difference between two types of intervention: psychotherapy with and without medical treatment. The main psychotherapeutic approaches adopted by rehabilitation facilities were psychological group therapy, individual psychological therapy (if necessary), educational intervention, and different forms of expressive activity. We found that subjects also on pharmacotherapy added to the psychotherapy program had higher scores in anxiety, depression, and mainly in psychological distress. They also showed less well-being and positive change. There were no significant differences

Table 2 Mean score, median, and the interquartile range (IQR) of CBA-OE scales at admission and discharge; score range between admission and discharge, in the total sample of patients

\begin{tabular}{|c|c|c|c|c|c|c|c|}
\hline \multicolumn{4}{|l|}{ Admission } & \multicolumn{4}{|l|}{ Discharge } \\
\hline CBA-OE scales & Mean (median) & IQR & $Z$ & Mean (median) & IQR & $\mathbf{Z}$ & $\Delta$-score \\
\hline Anxiety & $22.77(23)$ & $13-32$ & 0.56 & $14.39(13)$ & $7-20$ & -0.31 & -8.38 \\
\hline Well-being & $25.37(25)$ & $18-33$ & -0.34 & $34.12(34)$ & $28-40$ & 0.65 & +8.75 \\
\hline Positive change & $23.13(23)$ & $19-28$ & 0.30 & $27.31(28)$ & $23-32$ & 1.03 & +4.18 \\
\hline Depression & $26.8 \mathrm{I}(26)$ & $15-37$ & 0.17 & $15.49(14)$ & $8-20$ & 0.09 & -11.32 \\
\hline Psychological distress & $25.28(24)$ & $12-37$ & 0.80 & $14.76(1 \mathrm{I})$ & $7-20$ & -0.08 & -10.52 \\
\hline
\end{tabular}

Notes: Score range: anxiety 0-56; well-being 0-60; positive change 44-0; depression 0-76; psychological distress 0-84.

Abbreviations: CBA-OE, Cognitive Behavioral Assessment - Outcome Evaluation; Z, z score; $\Delta$-score, difference between admission score and discharge. 


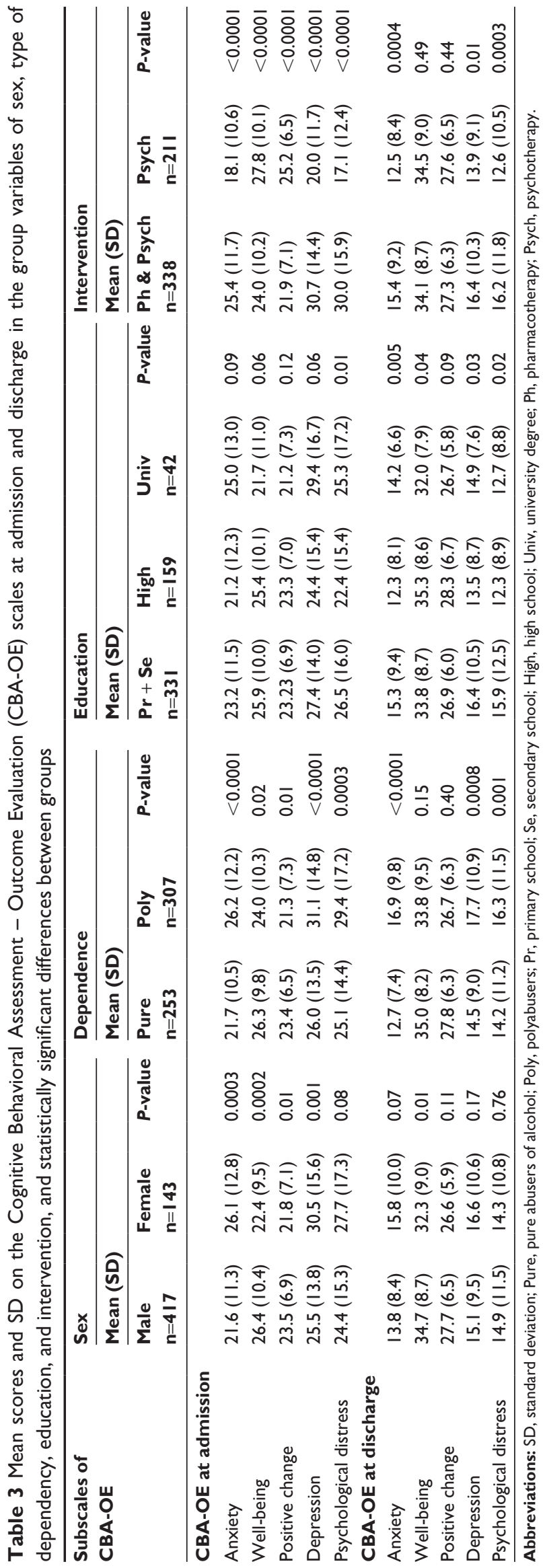

Table 4 Statistical differences on Cognitive Behavioral Assessment - Outcome Evaluation (CBA-OE) scales by Wilcoxon test between admission and discharge

\begin{tabular}{llll}
\hline CBA-OE scales & \multicolumn{2}{c}{ Wilcoxon signed-rank test } & Cohen's d \\
\cline { 2 - 3 } & Z-value & P-value & \\
\hline Anxiety & -15.009 & $<0.000 \mathrm{I}$ & $-0.7 \mathrm{I}$ \\
Well-being & -15.235 & $<0.000 \mathrm{I}$ & 0.85 \\
Positive change & $-1 \mathrm{I} .632$ & $<0.000 \mathrm{I}$ & 0.59 \\
Depression & -15.652 & $<0.000 \mathrm{I}$ & -0.78 \\
Psychological distress & -14.169 & $<0.000 \mathrm{I}$ & -0.66 \\
\hline
\end{tabular}

Notes: d-effects: small, $\geq 0.20$; medium, $\geq 0.50$; large, $\geq 0.80$.

between groups at discharge with regard to well-being and sense of a positive change, while they remained significant for the other psychological variables, though to a lesser extent. At the 6-month follow-up, 467 patients (80\%) answered, of which 318 (68\%) were still abstinent, 51 (11\%) had reduced their alcohol consumption, and only $98(21 \%)$ had started drinking again.

\section{Discussion}

The primary outcome of the study was the improvement in psychological functioning of patients who had completed residential treatment. The results of the CBA-OE questionnaire suggest the model of residential treatment for alcohol detoxification is effective. Indeed, several studies confirm that residential facilities offer a rehabilitation opportunity to manage multiple needs of these patients according to psychological, medical, and educational aspects, ${ }^{8,18,19}$ and that choosing abstinence as a goal results in superior outcome. ${ }^{20}$ Psychological health is an integral part of the recovery process, in view of the frequent coexistence of anxiety and depression in alcohol abusers. ${ }^{21,22}$ This study confirms the results of another study that used measures of quality of life $\mathrm{e}^{23}$ to assess the ability of patients to function in physical, familial, social, marital, and professional contexts. In fact, quality of life tends to improve after detoxification and treatment and worsen during relapse. The improvement in psychological functioning assessed through the five scales of the CBA-OE measures the changes in detail at the psychological level, and they could be the precursors for improvement in various aspects of the patient's life. The scores obtained on the scales of anxiety, depression, and psychological distress were all diminished. These results are in agreement with the current literature that shows that negative effects, including depressive states, decrease as a result of withdrawal treatment. ${ }^{24}$ Depression normally remits after 2 weeks of detoxification and abstinence, and falls to normal ranges within 3 weeks. That is, a large decrease in depression was observed in studies 
among alcohol-dependent patients during the protracted withdrawal and detoxification program, along with a decrease in self-reported craving. ${ }^{7,25}$ This may imply that depression in alcohol dependence is often as a result of the effects of chronic alcohol intoxication. ${ }^{26,27}$ In the same way, anxiety disorders and in particular social anxiety symptoms significantly decrease following alcohol detoxification. ${ }^{28,29}$ The scores on the well-being and positive changes scales attest to improvements in the measurements of self-perception of a positive change after the detoxification period. Some studies on life satisfaction showed that this variable increases over time in the recovery of alcohol-dependent inpatients and also quality of life shows a gradual improvement that may be linked to an increase in life stability. ${ }^{30,31}$

The CBA-OE questionnaire has proved to be an effective and useful tool to measure the psychological variables of recovery. It was also effective in the assessment of psychological treatment and to assess functioning in recovering problem drinkers. The differences between pure abusers and polyabusers on the scores of the CBA-OE at admission and discharge confirmed that the polyabuser group at admission had the highest values on the scales of anxiety, depression, and psychological distress, and consequently the lowest values on the scales of well-being and positive change. These results could be an expression of a major psychological impairment in the polyabuser group. This group included alcohol-dependent patients also addicted to other substances, and often we found a psychiatric comorbidity. The higher scores in this group compared to the pure abusers at discharge on the scales of anxiety, depression, and psychological distress were compatible with other studies, where polyabusers obtained scores on questionnaires assessing depression more similar to mental illness. ${ }^{32}$ The literature indicates that individuals who use alcohol or other drugs to relieve their depressive symptoms may require treatment of depression to achieve full remission after alcohol-disorder treatment and that their primary diagnosis was major depression. ${ }^{33}$ Furthermore, polyabusers are more impulsive than those who depend on one substance only. ${ }^{34}$ A study showed that high impulsivity, cluster B personality disorder, and polysubstance abuse were associated with early relapse. ${ }^{35}$ Polydrug abuse also increases the likelihood of overdose and suicide ${ }^{36}$ and poor treatment outcomes. ${ }^{37}$

In relation to sex, our data confirmed the sex-related differences in alcoholism, in particular before the detoxification treatment. In fact, we found that women had the worst scores on the scales of anxiety and depression and had lower outcomes on the scales of well-being and positive change at admission to residential facilities. These sex-related differences are in accordance with the acknowledged greater presence of significant depressive symptomatology in alcoholic women. ${ }^{38}$ Women are more likely to meet criteria for mood and anxiety disorders, ${ }^{39}$ but alcoholic women present a better prognosis, probably due to their better adherence to group therapies. ${ }^{40}$ At discharge from the period of detoxification treatment, we found significant differences between males and females only on the scale of well-being, with a lower score for women.

Another variable evaluated was the difference in levels of education and the psychological variables during the program of alcohol withdrawal. Our study showed that a lower level of education was associated with higher levels of psychological distress, and at the end of the residential treatment these subjects had higher levels of anxiety and depression. A different outcome was found on the scale of well-being at discharge: individuals who had a university education had lower scores than those with only a high school education. There has not been extensive research examining the influence of education on drinking behavior. In part, this aspect is related to cultural differences of different countries. For example, an Indian study showed the need of intervention on less educated individuals having high work stress and the need to promote awareness regarding the consequences of alcohol consumption on an individual's life. ${ }^{41}$ In Russia, education and amenities were inversely associated with alcohol-related problems. ${ }^{42}$ Another study on American college students asserted that social stress experienced by upper-class students may result in substance abuse to cope with the social pressure. ${ }^{43}$ Considering the risk factors related to alcohol use, social status offers a more complete understanding of the development of alcohol dependence, linked to the level of education. ${ }^{44}$

In addition, we used the CBA-OE to compare two therapeutic strategies used in residential facilities. The results showed more elevated symptoms of anxiety, depression, and distress in the group treated pharmacologically. This implies that the subjects to whom drugs were administered (drug therapy for withdrawal syndrome, craving, or mental health symptoms) were those who had more severe symptoms at admission, and this would be consistent with the choice of intervention that was adopted. Alcohol-use disorders (especially alcohol dependence) have demonstrated a strong association with mood disorders, in particular with anxiety disorders and depression. ${ }^{45-47}$ Moreover, alcohol-dependent patients with comorbid anxiety disorders exhibit increased levels of withdrawal symptoms during alcohol detoxification, 
and this comorbidity may complicate the treatment of alcohol dependence and its outcome. ${ }^{48,49}$ These patients may therefore require additional medical and psychological attention. ${ }^{4,26,50}$

A strong correlation was found between the severity of depression and the intensity of abstinence symptoms during hospitalization, ${ }^{51}$ and the relationship between depression and craving may explain why those individuals who were still depressed after alcohol detoxification and rehabilitation received antidepressants in order to reduce the probability of relapse. ${ }^{26,33}$ In our study, both pharmacological and psychological interventions were effective, especially in the recovery of the dimension of well-being and in the capacity of change in patients with more severe psychological symptoms. This is consistent with findings that heavy drinkers may enter treatment with higher motivation to change, perhaps because the severity of the patient's alcohol problems may enhance internal motivation to change, resulting in less ambivalence about treatment. ${ }^{52}$

Finally, the correlation between age and the psychological variables in alcohol-dependent patients during the rehabilitation treatment showed that younger subjects in our sample were those with higher levels of anxiety and distress, but with a higher probability at discharge to achieve a greater sense of well-being and positive change.

\section{Conclusion}

In this study, we evaluated psychological changes during a residential detoxification-treatment program with a short questionnaire that measures important psychological aspects related to alcohol dependence. The changes in anxiety disorders and depression in the current study are comparable to those in the literature. However, even if some of these disorders remit after abstinence, it still appears important to assess symptoms of anxiety and depression during the initial abstinence period, given their relevance for craving risk and early relapse. ${ }^{20,44}$

A limitation of the study is its retrospective design, and for this reason it was impossible to control some variables in order to obtain more information. In addition, it should be taken into account that the follow-up to 6 months is a relatively short period, and the results of interviews were based on responses from patients. However, the fairly large sample size of 560 hospitalized patients and the interesting association between sociodemographic characteristics and alcohol dependence in an Italian setting should be considered strong aspects of the study. In conclusion, the observation of a high risk of relapse for patients who suffer from a comorbid alcohol problem and a mood disorder suggests that a psychological outcome evaluation should be included in residential detoxificationtreatment programs, as it could be useful in identifying those subjects needing further psychosocial support after discharge.

\section{Acknowledgments}

The CBA-OE authors would like to express their very great appreciation to COordinamento Residenzialità Riabilitative ALcologiche (CORRAL): G Cerizza, F Girardi, A Jaretti Sodano, L Liberto, D Mioni, P Ranaletti, and P Vizzuso.

\section{Disclosure}

The authors report no conflicts of interest in this work.

\section{References}

1. Volpicelli JR. Alcohol abuse and alcoholism: an overview. J Clin Psychiatry. 2001;62 Suppl 20:4-10.

2. Yang MJ. The Chinese drinking problem: a review of literature and its implication in a cross-cultural study. Kaohsiung J Med Sci. 2002; 18(11):543-550

3. Dev R, Parsons HA, Palla S, Palmer JL, Del Fabbro E, Bruera E. Undocumented alcoholism and its relationship with tobacco and illegal drug use in advanced cancer patients. Cancer. 2011;117(19):4551-4556.

4. Liu Y, Nguyen N, Colditz GA. Links between alcohol consumption and breast cancer: a look at the evidence. Womens Health (Lond Engl). 2015;11(1):65-77.

5. Santovito A, Cervella P, Delpero M. Evidence of genotoxicity in lymphocytes of non-smoking alcoholics. Mol Biol Rep. 2015;42(1):53-59.

6. Fuller RK, Hiller-Sturmhöfel S. Alcoholism treatment in the United States. An overview. Alcohol Res Health. 1999;23(2):69-77.

7. Hayashida M. An overview of outpatient and inpatient detoxification. Alcohol Health Res World. 1998;22(1):44-46.

8. Zambon A, Soares Pinto SP, Agostini D, et al. [Alcohol-addiction inpatient: characteristics of patients and rehabilitation program]. Minerva Med. 2013;104(2):193-206. Italian.

9. Anderson DJ, McGovern JP, DuPont RL. The origins of the Minnesota model of addiction treatment - a first person account. $J$ Addict Dis. 1999;18(1):107-114.

10. Hodolin V, Corlito G. Psichiatria, Alcologia, Riabilitazione. Trento, Italy: Centro Studi Erickson; 1996.

11. Hinnental I, Cibin M. Il Trattamento Residenziale Breve delle Dipendenze da Alcol e Cocaina: Il Modello Soranzo. Turin: SEED; 2011.

12. Baker TB, Piper ME, McCarthy DE, Majeskie MR, Fiore MC. Addiction motivation reformulated: an affective processing model of negative reinforcement. Psychol Rev. 2004;111(1):33-51.

13. Sinha R, Fox HC, Hong KI, Hansen J, Tuit K, Kreek MJ. Effects of adrenal sensitivity, stress- and cue-induced craving, and anxiety on subsequent alcohol relapse and treatment outcomes. Arch Gen Psychiatry. 2011;68(9):942-952.

14. American Psychiatric Association. Diagnostic and Statistical Manual of Mental Disorders. 4th ed. Washington: American Psychiatric Association; 1994.

15. Bertolotti G, Michielin P, Vidotto G, et al. Metric qualities of the cognitive behavioral assessment for outcome evaluation to estimate psychological treatment effects. Neuropsychiatr Dis Treat. 2015;11:2449-2460.

16. Michielin P, Vidotto G, Altoè G, et al. [New questionnaire to evaluate psychological treatment effects]. G Ital Med Lav Ergon. 2008; 30(1 Suppl A):A98-A104. Italian.

17. Cohen J. Statistical Power Analysis for the Behavioral Sciences. New Jersey: LEA Publishers; 1988. 
18. Cerizza G, Ranalletti MP. Residential treatment as a rehabilitation opportunity for alcoholism care. Open Toxicol J. 2013;6 Suppl 1(M5): 34-37.

19. Rundio A Jr. Implementing an evidence-based detoxification protocol for alcoholism in a residential addictions treatment facility. Nurs Clin North Am. 2013;48(3):391-400.

20. Heather N, Adamson SJ, Raistrick D, Slegg GP. Initial preference for drinking goal in the treatment of alcohol problems: I. Baseline differences between abstinence and non-abstinence. Alcohol Alcohol. 2010;45(2):128-135.

21. Wetterling T, Junghanns K. Psychopathology of alcoholics during withdrawal and early abstinence. Eur Psychiatry. 2000;15(8):483-488.

22. Hibbert LJ, Best DW. Assessing recovery and functioning in former problem drinkers at different stages of their recovery journeys. Drug Alcohol Rev. 2011;30(1):12-20.

23. Picci RL, Oliva F, Zuffranieri M, et al. Quality of life, alcohol detoxification and relapse: is quality of life a predictor of relapse or only a secondary outcome measure? Qual Life Res. 2014;23(10):2757-2767.

24. Andersohn F, Kiefer F. Depressive mood and craving during alcohol withdrawal: association and interaction. Ger J Psychiatr. 2004; 7(2):6-11.

25. de Sousa Uva MC, de Timary P, Cortesi M, Mikolajczak M, du Roy de Blicquy $\mathrm{P}$, Luminet $\mathrm{O}$. Moderating effect of emotional intelligence on the role of negative affect in the motivation to drink in alcoholdependent subjects undergoing protracted withdrawal. Pers Individ Dif. 2010;48(1):16-21.

26. Kuria MW, Ndetei DM, Obot IS, et al. The association between alcohol dependence and depression before and after treatment for alcohol dependence. ISRN Psychiatry. 2012;2012:482802.

27. Brown SA, Schuckit MA. Changes in depression among abstinent alcoholics. J Stud Alcohol. 1988;49(5):412-417.

28. Liappas J, Paparrigopoulos T, Tzavellas E, Christodoulou G. Alcohol detoxification and social anxiety symptoms: a preliminary study of the impact of mirtazapine administration. $J$ Affect Disord. 2003;76(1-3):279-284.

29. Gorwood P. [Alcohol dependence and anxious disorders: dangerous liaisons]. Rev Prat. 2010;60(6):801-806. French.

30. Laudet AB, Morgen K, White WL. The role of social supports, spirituality, religiousness, life meaning and affiliation with 12-step fellowships in quality of life satisfaction among individuals in recovery from alcohol and drug problems. Alcohol Treat Q. 2006;24(1-2):33-73.

31. Marini M, Schnornberger TM, Brandalise GB, Bergozza M, Heldt E. Quality of life determinants in patients of a psychosocial care center for alcohol and other drug users. Issues Ment Health Nurs. 2013; 34(7):524-530.

32. Cangemi S, Giorgi I, Bonfiglio NS, Renati R, Vittadini G. Impulsiveness and time perception in alcohol dependent patients in alcoholic rehabilitation treatment. G Ital Med Lav Ergon. 2010;32(3 Suppl B):B23-B28.

33. Boden JM, Fergusson DM. Alcohol and depression. Addiction. 2011; 106(5):906-914.

34. O'Boyle M, Barratt ES. Impulsiveness and DSM-III-R personality disorders. Pers Individ Dif. 1993;14(4):609-611.

35. Curran GM, Booth BM. Longitudinal changes in predictor profiles of abstinence from alcohol use among male veterans. Alcohol Clin Exp Res. 1999;23(1):141-143.

Neuropsychiatric Disease and Treatment

\section{Publish your work in this journal}

Neuropsychiatric Disease and Treatment is an international, peerreviewed journal of clinical therapeutics and pharmacology focusing on concise rapid reporting of clinical or pre-clinical studies on a range of neuropsychiatric and neurological disorders. This journal is indexed on PubMed Central, the 'PsycINFO' database and CAS,
36. Risser D, Schneider B. Drug related deaths between 1985 and 1992 examined at the Institute of Forensic Medicine in Vienna, Austria. Addiction. 1994;89(7):851-857.

37. Rounsaville BJ, Dolinsky ZS, Babor TF, Meyer RE. Psychopathology as a predictor of treatment outcome in alcoholics. Arch Gen Psychiatry. 1987; 44(6):505-513.

38. Travaglini R, Giardinelli L. [Alcoholism and sex difference]. G Ital Psicopatol. 2005;11(4):437-444. Italian.

39. Conway KP, Compton W, Stinson FS, Grant BF. Lifetime comorbidity of DSM-IV mood and anxiety disorders and specific drug use disorders: results from the National Epidemiologic Survey on Alcohol and Related Conditions. J Clin Psychiatry. 2006;67(2):247-257.

40. Arnau MM. [Differential features of female alcoholism]. Adicciones. 2010;22(4):339-352. Spanish.

41. Vignesh BT, Singh AK, Mohan SK, Murthy S, Joshi A. Association between socio-demographics and alcohol dependence among individuals living in an Indian setting. Glob J Health Sci. 2014;6(3):16-26.

42. Cook S, De Stavola B, Saburova L, et al. Socio-demographic predictors of dimensions of the AUDIT score in a population sample of workingage men in Izhevsk, Russia. Alcohol Alcohol. 2011;46(6):702-708.

43. Luthar SS. The culture of affluence: psychological costs of material wealth. Child Dev. 2003;74(6):1581-1593.

44. Harrell ZA, Huang JL, Kepler DM. Brief report: Affluence and college alcohol problems - the relevance of parent- and child-reported indicators of socioeconomic status. J Adolesc. 2013;36(5):893-897.

45. Hasin DS. Introduction to DSM-5 criteria linked papers in drug and alcohol dependence. Drug Alcohol Depend. 2012;122(1-2):20-21.

46. Moreno C, Hasin DS, Arango C, et al. Depression in bipolar disorder versus major depressive disorder: results from the National Epidemiologic Survey on Alcohol and Related Conditions. Bipolar Disord. 2012;14(3):271-282.

47. Lechner WV, Shadur JM, Banducci AN, Grant DM, Morse M, Lejuez CW. The mediating role of depression in the relationship between anxiety sensitivity and alcohol dependence. Addict Behav. 2014; 39(8):1243-1248.

48. Bradizza CM, Stasiewicz PR, Paas ND. Relapse to alcohol and drug use among individuals diagnosed with co-occurring mental health and substance use disorders: a review. Clin Psychol Rev. 2006;26(2): $162-178$.

49. Marquenie LA, Schadé A, Van Balkom AJ, et al. Comorbid phobic disorders do not influence outcome of alcohol dependence treatment. Results of a naturalistic follow-up study. Alcohol Alcohol. 2006;41(2): 168-173.

50. Schellekens AF, de Jong CA, Buitelaar JK, Verkes RJ. Co-morbid anxiety disorders predict early relapse after inpatient alcohol treatment. Eur Psychiatry. 2015;30(1):128-136.

51. Francke ID, Viola TW, Tractenberg SG, Grassi-Oliveira R. Childhood neglect and increased withdrawal and depressive severity in crack cocaine users during early abstinence. Child Abuse Negl. 2013;37(10): 883-889.

52. Penberthy JK, Hook JN, Vaughan MD, et al. Impact of motivational changes on drinking outcomes in pharmacobehavioral treatment for alcohol dependence. Alcohol Clin Exp Res. 2011;35(9):1694-1704.

\footnotetext{
Submit your manuscript here: http://www.dovepress.com/neuropsychiatric-disease-and-treatment-journal
}

and is the official journal of The International Neuropsychiatric Association (INA). The manuscript management system is completely online and includes a very quick and fair peer-review system, which is all easy to use. Visit http://www.dovepress.com/testimonials.php to read real quotes from published authors. 